\title{
Controlling the formation and stability of ultra-thin nickel silicides - an alloying strategy for preventing agglomeration
}

\author{
F.A. Geenen ${ }^{\mathrm{a}}$, K. van Stiphout ${ }^{\mathrm{b}}$, A. Nanakoudis ${ }^{\mathrm{c}}, \mathrm{S} . \mathrm{Bals}^{\mathrm{c}}$, A. Vantomme ${ }^{\mathrm{b}}, \mathrm{J}$.

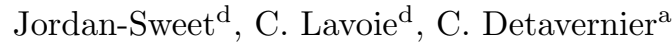 \\ ${ }^{a}$ Department of Solid-State Sciences, Ghent University, 9000 Gent, Belgium \\ ${ }^{b}$ Instituut voor Kern- en Stralingsfysica, KU Leuven, B-3001 Leuven, Belgium \\ ${ }^{c}$ EMAT, University of Antwerp, Groenenborgerlaan 171, B-2020 Antwerp, Belgium \\ ${ }^{d} I B M$ T.J. Watson Research Center, Yorktown Heights, NY, USA
}

\begin{abstract}
The electrical contact of the source and drain regions in state-of-the-art CMOS transistors is nowadays facilitated through $\mathrm{NiSi}$, which is often alloyed with $\mathrm{Pt}$ in order to avoid morphological agglomeration of the silicide film. However, the solid-state reaction between as-deposited $\mathrm{Ni}$ and the $\mathrm{Si}$ substrate exhibits a peculiar change for as-deposited Ni films thinner than a critical thickness of $t_{c}=5 \mathrm{~nm}$. Whereas thicker films form polycrystalline NiSi upon annealing above $450{ }^{\circ} \mathrm{C}$, thinner films form epitaxial $\mathrm{NiSi}_{2}$ films which exhibit a high resistance towards agglomeration. For industrial applications, it is therefore of utmost importance to assess the critical thickness with high certainty and find novel methodologies to either increase or decrease its value, depending on the aimed silicide formation. This paper investigates $\mathrm{Ni}$ films between 0 and $15 \mathrm{~nm}$ initial thickness by using of 'thickness gradients', which provide semi-continuous information on silicide formation and stability as a function of as-deposited layer thickness. The alloying of these Ni layers with $10 \%$ Al, Co, Ge, Pd or Pt renders a significant change in the phase sequence as a function of thickness and dependent on the alloying element. The addition of these ternary impurities therefore change the critical thickness $t_{c}$. The results are discussed in the framework of
\end{abstract}

Email address: christophe.detavernier@ugent.be (C. Detavernier) 
classical nucleation theory.

Keywords: nickel silicide, epitaxy, axiotaxy, texture

\section{Introduction}

Metal silicides are used in micro-electronics to establish low-resistance contacts to the source and drain regions of Si-based transistors. The currently preferred material, NiSi, exhibits limited morphological stability and suffers from 5 significant agglomeration upon annealing (e.g. $\left.>500^{\circ} \mathrm{C}\right)$ [1, 2, 3, 4. Moreover, $\mathrm{NiSi}$ transforms into $\mathrm{NiSi}_{2}$ at higher temperature (e.g. $>700^{\circ} \mathrm{C}$ ), inducing significant change in silicon consumption and electrical properties such as contact resistance and sheet resistivity. The addition of small amounts of Pt (e.g. 10 at.\%) is currently being used to delay both the agglomeration process and high-temperature $\mathrm{NiSi}_{2}$ transformation [5]. Because agglomeration is driven by surface and interface energy, it is significantly more difficult to avoid for thinner silicide films. 6, 7]. This can partly be compensated by incorporating a higher Pt concentration, but this results in a higher parasitic resistivity and only delays agglomeration instead of completely stabilising the morphology. Therefore, there is an ongoing interest in alternative contact materials with improved morphological stability, for such thin (e.g. $<10 \mathrm{~nm}$ ) layers.

Pioneering work by Tung et al. 8] demonstrated that the deposition of sub$10 \mathrm{~nm}$ thin Ni films onto Si substrates can result in a completely different silicide phase sequence, which forms epitaxial $\mathrm{NiSi}_{2}$ instead of $\mathrm{NiSi}$ at low temperature. Recently, the effect of this ultrathin phase formation has regained interest 9, 10, 11, as the industry is evolving towards sub-10 nm silicide thicknesses. These studies indicate that there exists a well-defined boundary between the 'regular' regime - where reaction of e.g. $10 \mathrm{~nm} \mathrm{Ni}$ at $450{ }^{\circ} \mathrm{C}$ is known to result in a polycrystalline NiSi film with axiotaxial texture - and the 'ultrathin' regime where reaction of e.g. $3 \mathrm{~nm} \mathrm{Ni}$ at similar temperatures results in the formation of an epitaxial $\mathrm{NiSi}_{2}$ layer. De Keyser et al. 9] showed that the ultrathin phase regime (i.e. below the critical thickness) exhibits very high morphological 
stability. Knoll et al. [12, 13] furthermore reported on the beneficial effect of the lower Schottky barrier height of these epitaxial $\mathrm{NiSi}_{2}$ films.

As the silicide contacts in the state-of-the-art micro-electronics industry are trending to sub-10 nm thickness values, it is important to determine and control the exact value of the critical thickness which differentiates these two phase-formation regimes. Gao et al. [14] recently reported on a biased-sputterdeposition strategy with pure $\mathrm{Ni}$ films, which increases the as-deposited mixed

$35 \mathrm{Ni} / \mathrm{Si}$ interface region and increases the maximum thickness of the formed epitaxial $\mathrm{NiSi}_{2}$ films, thus increasing the critical thickness.

This paper discusses an alternative strategy based on the incorporation of ternary elements in the as-deposited film, very similar to the current approach of using Pt-alloying to enhance the performance of NiSi films. The effective

40 value of the critical thickness $t_{c}$ is determined through a methodology based on the deposition of a nickel film on a $\mathrm{Si}(001)$ wafers, where the nickel thickness is dependent on the position of the wafer. These deposited wafers can be used as combinatorial thickness libraries to assess $t_{c}$ with high accuracy as explained in the experimental section. We studied the critical thickness for unalloyed $\mathrm{Ni}$

45 films, as well as films alloyed with 10 at \% of a ternary element (i.e. Al, Co, Ge, $\mathrm{Pd}, \mathrm{Pt})$.

\section{Experimental methods}

Ni films were deposited on $150 \mathrm{~mm}$ diameter $\mathrm{Si}(001)$ and silicon-on-insulator (SOI) substrates through physical vapour deposition with a combinatorial 50 approach. The thickness gradient was achieved by modulating the amount of deposited $\mathrm{Ni}$ with a triangular-shaped shadow mask, which resulted in a high deposition rate at one side of the substrate while almost eliminating the deposition rate at the other side of the wafer. The final deposited Ni gradient can be described as a thickness library, where the deposited Ni thickness varies quasi-linearly in the direction of the gradient as a function of the position on the substrate. The $\mathrm{Ni}$ content of the gradient was determined to vary from 
0.5 to $15 \mathrm{~nm}$ equivalent thickness as determined through Rutherford backscattering spectrometry (RBS) measurements, and thus covers the thickness region of interest to study the critical thickness in the Ni-Si phase formation. This combinatorial approach is not only resource and time efficient, but also ensures that identical experimental conditions were applied for all $\mathrm{Ni}$ thickness values of interest.

The substrates received standard (Radio Corporation of America, RCA) chemical cleaning, ending with a $20 \mathrm{~s}$ dip in a $3 \%$ HF solution prior to loading into the vacuum chamber. Sputter deposition was performed in an $\mathrm{Ar}$ atmosphere of $5 \times 10^{-3}$ mbar, after first reaching a background pressure of $6 \times 10^{-7}$ mbar. The Ni thickness gradient was optionally alloyed with $10 \pm 2$ at.\% Al, Co or Pt by co-sputtering. This was obtained through a second shadow mask for the alloy which resulted in a constant ratio between the Ni and alloy signals as verified through X-Ray Fluorescence (Co, Pt) and RBS ( $\mathrm{Al}$ ) along the thickness gradient. Ni films with 10 at.\% of Pd and Ge were also deposited with discrete equivalent $\mathrm{Ni}$ thicknesses (i.e. without thickness gradient) of 2, 3, 6 and $9 \mathrm{~nm}$ and are discussed supplementary to the gradient datasets.

These unalloyed and alloyed thickness gradients are compared as function of their Ni content. The alloying species are added in addition to the original $\mathrm{Ni}$ thickness gradient, and therefore the total thickness of the as-deposited layer is higher for the alloyed films. As $\mathrm{Co}$ and $\mathrm{Pt}$ (and $\mathrm{Pd}$ ) are known to replace $\mathrm{Ni}$ atoms during silicide formation, the addition of these alloying elements render an effective increase in the metal supply to form $\mathrm{Ni}_{x} \mathrm{M}_{y} \mathrm{Si}_{z}(\mathrm{M}=\mathrm{Co}, \mathrm{Pt}, \mathrm{Pd})$ alloys. Other papers therefore prefer to keep the as-deposited total metal supply constant by reducing the Ni content when adding an alloying species. Such approach is not easily translated to $\mathrm{Al}$ (or $\mathrm{Ge}$ ) alloying, which is known to replace the Si atoms during silicide formation. For conciseness, the amount of nickel present at every position on each gradient will be referred to by using the equivalent $\mathrm{Ni}$ thickness of the gradient, i.e. the as-deposited Ni thickness which would be measured when no alloy would be added to the layer.

The thickness library was then used in two complementary experimental ap- 
proaches. In a first approach, a $150 \mathrm{~mm}$ long strip from the thickness library was annealed up to a specific temperature and its properties were characterised after quenching as a function of position within the library (i.e. as a function of as-deposited Ni thickness). Ex situ techniques were used, such as scanning electron microscopy (SEM) to evaluate layer morphology, pole-figure measurements to investigate crystalline phase, and texture and transmission electron microscopy (TEM) to assess roughness and local composition. In a second approach, a $150 \mathrm{~mm}$ long thickness gradient strip was cut into 15 smaller pieces of $\sim 10 \mathrm{~mm}$, resulting in a set of discrete samples with an average thickness difference of $1 \mathrm{~nm}$. Subsequently, these individual samples were studied with in situ techniques, which consisted of a continuous acquisition of the sheet resistance (SR) and X-ray diffraction (XRD) during annealing. To enable a straightforward comparison in the measurements acquired both in situ during annealing, and ex situ after annealing, we used the same annealing conditions.

In situ XRD was used to monitor the solid-state reaction as a function of temperature and was performed at the X20C beamline of the National Synchrotron Light Source (NSLS). The wavelength of the incidence X-rays was selected at $0.180 \mathrm{~nm}$ with an energy resolution of $1.5 \%$ by a multilayer monochromator. The diffraction pattern was monitored through a linear detector having an angular range of $14^{\circ}$ in $2 \theta$. These diffractograms are plotted as a function of temperature and diffraction angle using a linear gray-scale map for the recorded intensities, where darker represents a higher intensity.

Further identification of the formed phases is enabled through ex situ Xray pole figures, measured for samples quenched at $700{ }^{\circ} \mathrm{C}$. For recording such pole figures, the sample is tilted around both the in-plane and out-of-plane axes (denoted as the $\chi$ and $\phi$ angle, respectively), to obtain information on the preferential orientation of the probed crystalline planes. A polar plot of the 115 diffraction intensity then represents the distribution of the crystalline plane's preferred orientation, also denoted as the texture of the crystal grains. The pole figures reported in this work were acquired using a four-circle diffractometer at the X20A beamline of the NSLS, using X-rays with a wavelength of $\lambda=$ 
$0.154 \mathrm{~nm}$, and a custom linear detector covering 20 to $60^{\circ}$ in $2 \theta$, and by scanning the gradient indicates an abrupt boundary between two regions in the thickness library with a clear difference in color. This boundary is situated at a thickness of $t_{c}=4.8 \mathrm{~nm}$ (Fig. 1 $\mathrm{b}$ ) and SEM images in figure 1 $\mathrm{k}$ show that the color change is due to a different morphology of the sample. Films thicker than $t_{c}$ showed 145

In situ XRD confirms that the observed critical boundary $t_{c}$ is the demarcation line between the two thickness regimes that are described in the literature 
(a)

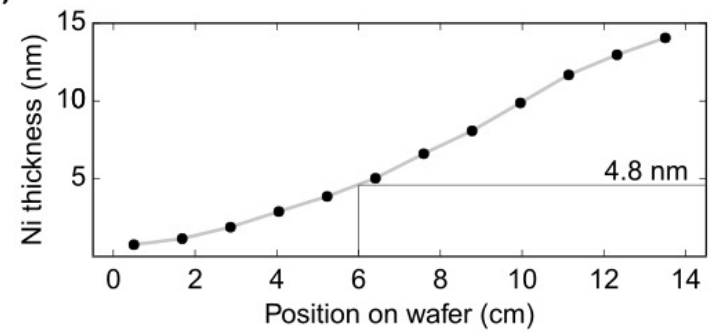

(b)

stable $\quad t_{c} \quad$ agglomerating

(c)

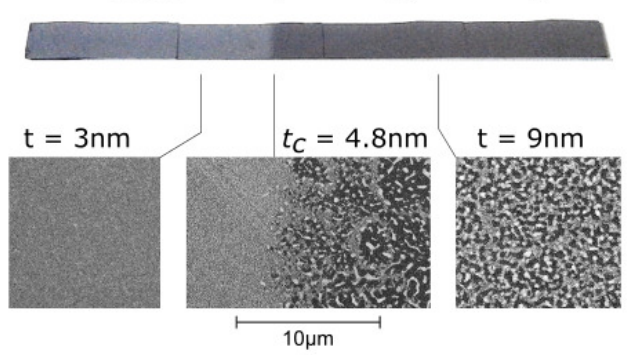

Figure 1: Thickness gradients were used to study the solid-state reaction between $\mathrm{Ni}$ and $\mathrm{Si}(001)$. (a) The equivalent Ni thickness was determined from RBS measurements, showing a quasi-linear dependence of de thickness with the position on the wafer. (b) Photographic picture of a $150 \mathrm{~mm}$ long strip from the thickness gradient after annealing to $700{ }^{\circ} \mathrm{C}$, exhibiting a clear color change at a Ni thickness of $t_{c}=4.8 \mathrm{~nm}$. (c) SEM micrographs conducted at several positions on the annealed gradient show a significant difference in morphology at $t_{c}$. 

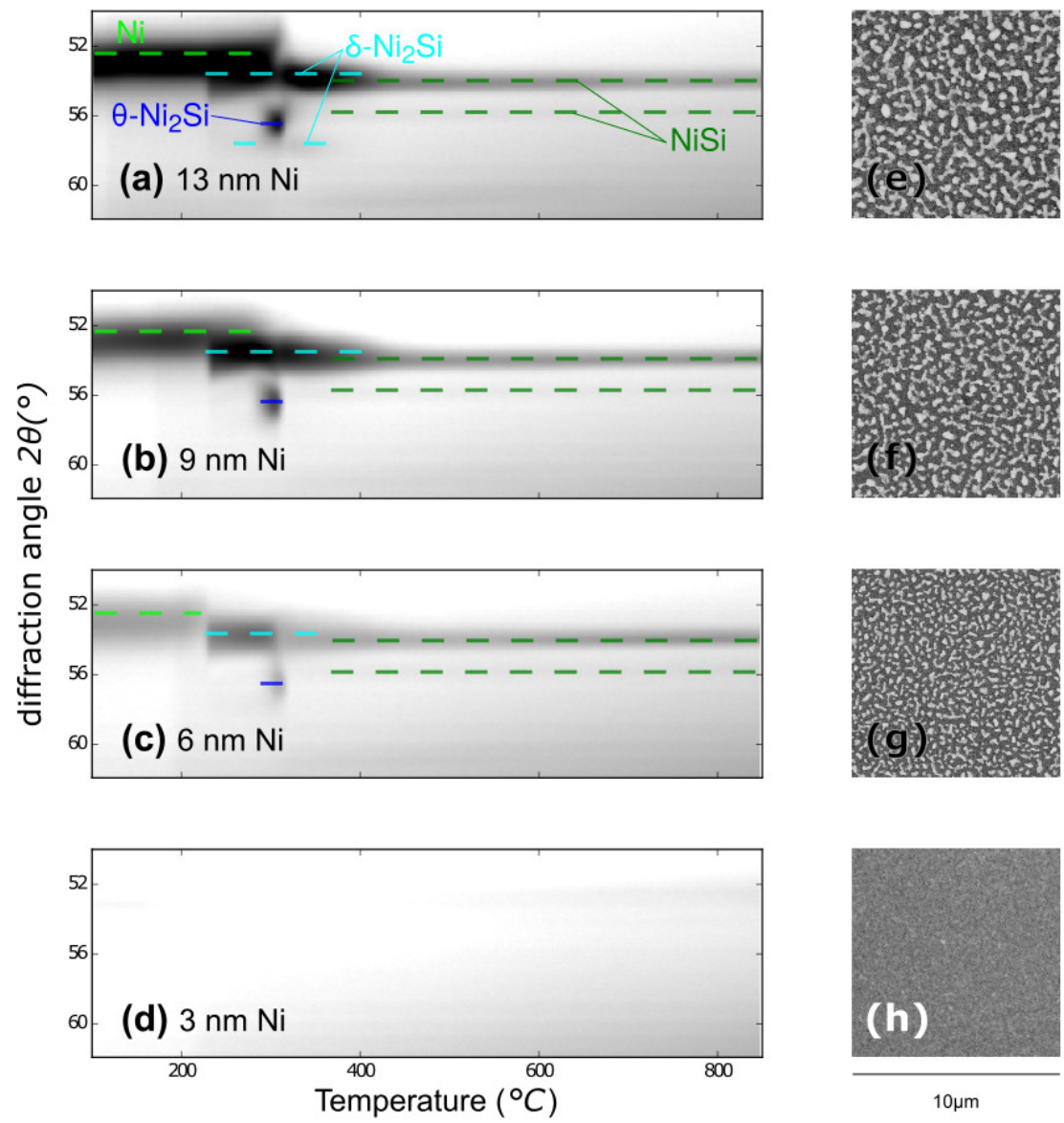

$10 \mu \mathrm{m}$

Figure 2: In situ XRD measurements during annealing indicate a significant difference in phase formation above and below the critical thickness $t_{c}=4.8 \mathrm{~nm}$. The sequence of several silicides is observed for films thicker than $t_{c}$, whereas thinner films do not show any diffraction, indicating the possible formation of epitaxial silicides. 
(a)

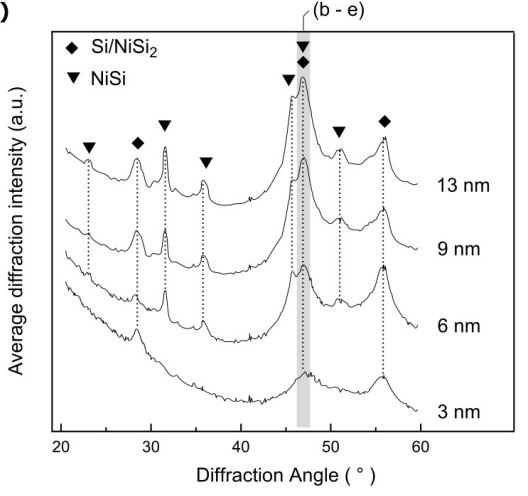

(b) $13 \mathrm{~nm}$

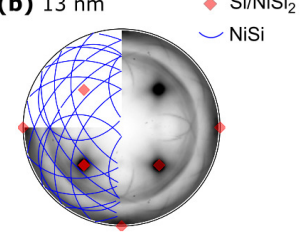

(d) $6 \mathrm{~nm}$

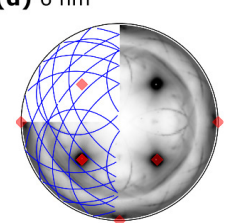

(c) $9 \mathrm{~nm}$

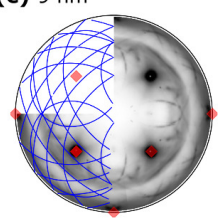

(e) $3 \mathrm{~nm}$

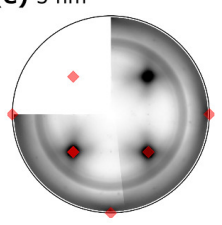

Figure 3: (a) Pole figures acquired from Ni samples quenched at $700{ }^{\circ} \mathrm{C}$ integrated in $\chi$ and $\phi$ result in a powder-like diffraction pattern, allowing unambiguous phase identification. Further investigation of the individual pole figure at $2 \theta \simeq 47.5^{\circ}$ (covering diffraction of $\mathrm{NiSi}(211) /(202)$ and $\mathrm{NiSi}_{2}(202)$ planes) shows the axiotaxial texture of the NiSi phase for 13 (b), 9 (c) and $6 \mathrm{~nm}$ (d) Ni. $3 \mathrm{~nm}$ (e) Ni instead only contains features originating from either the Si substrate and/or type-A epitaxial $\mathrm{NiSi}_{2}$.

[9, 10, 11. Indeed, samples above the critical thickness (e.g. 6, 9 and $13 \mathrm{~nm}$, Fig. 2a, b and c) show the well-known phase sequence which forms NiSi above $400{ }^{\circ} \mathrm{C}$, after first forming intermediate $\delta-\mathrm{Ni}_{2} \mathrm{Si}$ and transient $\theta-\mathrm{Ni}_{2} \mathrm{Si}$ [19]. However, in situ XRD from samples below the critical thickness (e.g. $3 \mathrm{~nm}$, Fig. 2 $\mathrm{d}$ ) do not show any clear diffraction signal from silicide phases formed during the anneal. We equate the absence of diffraction to the formation of epitaxial silicides. Due to the fixed geometry of the in situ XRD set-up, diffracting planes will only be observed if they are oriented (nearly) parallel to the substrate's surface. Consequently, an epitaxial phase does not necessarily diffract in the probed reciprocal space.

The sudden change in phase formation at the critical thickness is further corroborated by ex situ pole-figure measurements. Within these measurements, diffraction intensities are monitored while the sample is rotated both in- and out-of-plane (respectively angles $\phi$ and $\chi$ ), thus avoiding the issues related with a fixed geometry described above. As a first step in the pole-figure data analysis, one can use these measurements for unambiguous phase identification by 
integrating the observed intensity over all $\phi$ and $\chi$, for every value of $2 \theta$. This [8, 9, 10, 11 .

The observation of such a clear thickness-dependent effect through the use of combinatorial libraries illustrates the unique capability of these thickness 
(a)

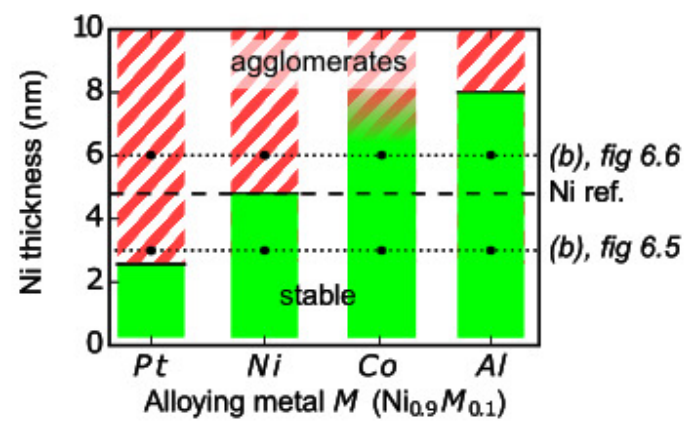

(b)

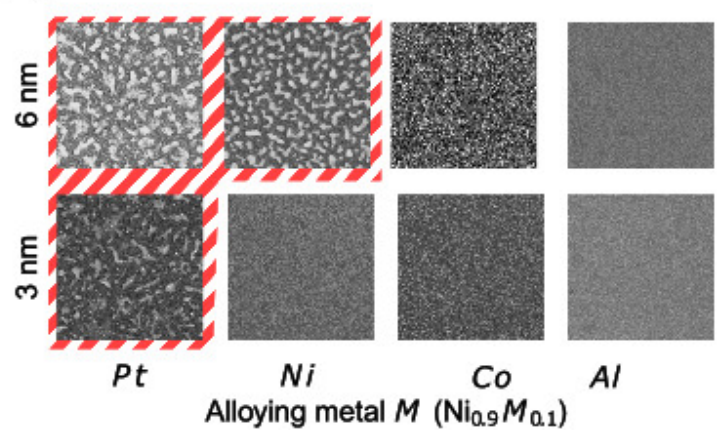

Figure 4: Unalloyed and alloyed (10 at.\% Al, Co and Pt) thickness gradients were quenched at $700^{\circ} \mathrm{C}$ in order to determine the critical thickness differences in morphological stability. Co-alloying shows a continuous increase in roughness as a function of Ni thickness.

gradients to investigate the critical thickness in the $\mathrm{Ni}$-Si solid-state reaction as a function of position.

\subsection{Effect of alloying on the critical thickness}

Thickness gradients were subsequently used to investigate the critical change in phase formation when ternary elements are included in the as-deposited layer.

Thickness gradients, alloyed with 10 at.\% Al, Co or Pt, were again quenched at $700^{\circ} \mathrm{C}$. Samples with $\mathrm{Al}$ or Pt show a similar critical change in morphology at positions equivalent to a $\mathrm{Ni}$ thickness of $t_{c}=8.0$ and $2.6 \mathrm{~nm}$, respectively (Fig. (4). The changes in equivalent thickness is not the consequence of a merely 
(a)

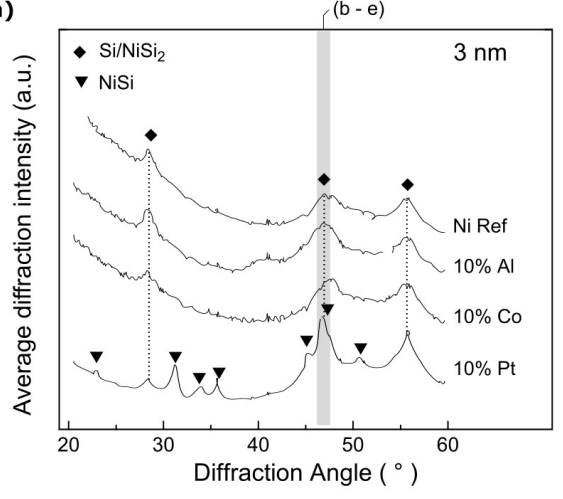

(b) Ni Ref

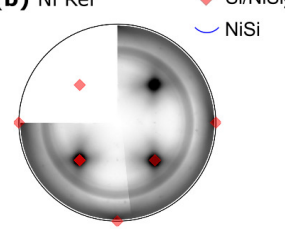

(d) $10 \% \mathrm{Co}$

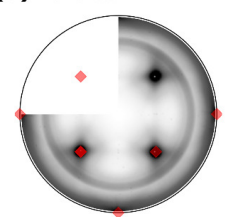

(c) $10 \% \mathrm{Al}$

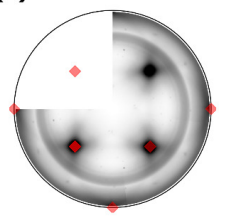

(e) $10 \% \mathrm{Pt}$

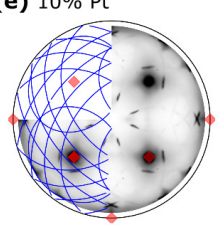

Figure 5: (a) The constructed powder-like diffraction patterns are used to identify the formed silicde after annealing $3 \mathrm{~nm}$ thick Ni films, which are alloyed with 10 at.\% $\mathrm{Al}$, Co or Pt, to $700^{\circ} \mathrm{C}$. The individual pole figures at $2 \theta \simeq 47.5^{\circ}$ exclude the occurence of any other silicide phase except type-A epitaxial $\mathrm{NiSi}_{2}$ being formed for an unalloyed $\mathrm{Ni}$ film (b), or when alloyed with $\mathrm{Al}$ (c) or Co (d). NiSi is clearly present when alloyed with Pt (e).

increased metal supply, e.g. the combination of $2.6 \mathrm{~nm} \mathrm{Ni}$ in addition to 10 at.\% Pt) is far less than the critical thickness $t_{c}=4.8 \mathrm{~nm}$ for unalloyed Ni films. When the gradient strip was alloyed with Co, no visual abrupt difference in morphology was observed as a function of thickness. SEM images (not shown) instead show an increased roughness for Ni thicknesses between 5 and $11 \mathrm{~nm}$, but do not show the severe agglomeration as observed for an unalloyed film.

Further investigation of these alloyed thickness gradients through ex situ pole figures confirms that the value of $t_{c}$ can indeed be varied through alloying. A selection of these measurements is displayed for 3 and $6 \mathrm{~nm}$ as-deposited $\mathrm{Ni}$ thickness in figures 5 and 6 , respectively. The simulated 'powder-like' diffraction patterns 5 and 6 a) includes diffraction intensities regardless of the diffraction plane's orientation, and unambiguously allows the identification of the crystalline phases present in the film. When $3 \mathrm{~nm}$ films are alloyed with $\mathrm{Al}$ or $\mathrm{Co}$, no significant differences are observed by comparing these measurements with those of an unalloyed Ni film. However, Pt alloyed samples now clearly contain NiSi diffraction. 

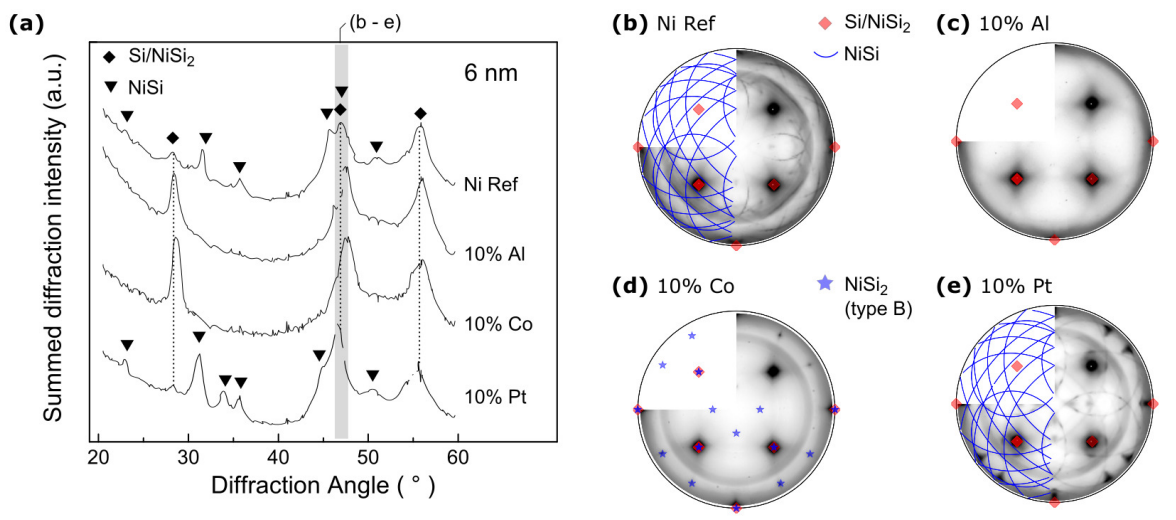

Figure 6: (a) The constructed powder-like diffraction patterns are used to identify the formed silicde after annealing $6 \mathrm{~nm}$ thick Ni films, which are alloyed with 10 at.\% Al, Co or Pt, to $700^{\circ} \mathrm{C}$. NiSi is clearly present for the unalloyed film (b), or when Pt is used as ternary element (e). The individual pole figures at $2 \theta \simeq 47.5^{\circ}$ confirm the absence of any silicide other than $\mathrm{NiSi}_{2}$ when 10 at.\% $\mathrm{Al}$ or Co are added.

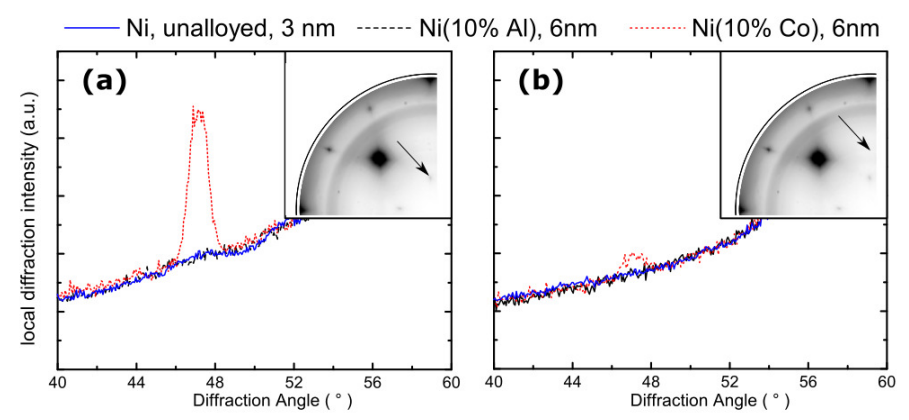

Figure 7: The diffraction intensities of a selection of samples for two specific orientations: (a) $\phi$ $=90^{\circ}$, and $\chi=90^{\circ}$, corresponding with type-B oriented $\mathrm{NiSi}_{2}(220)$ planes $\left(2 \theta=47.3^{\circ}\right)$, and (b) $\phi=90^{\circ}$, and $\chi=32^{\circ}$, corresponding with randomly-oriented $\mathrm{NiSi}_{2}(220)$ planes $\left(2 \theta=47.3^{\circ}\right)$. The insets illustrate the location of these coordinates on the $\mathrm{NiSi}_{2}(220)$ polefigure of the Co-alloyed sample. 

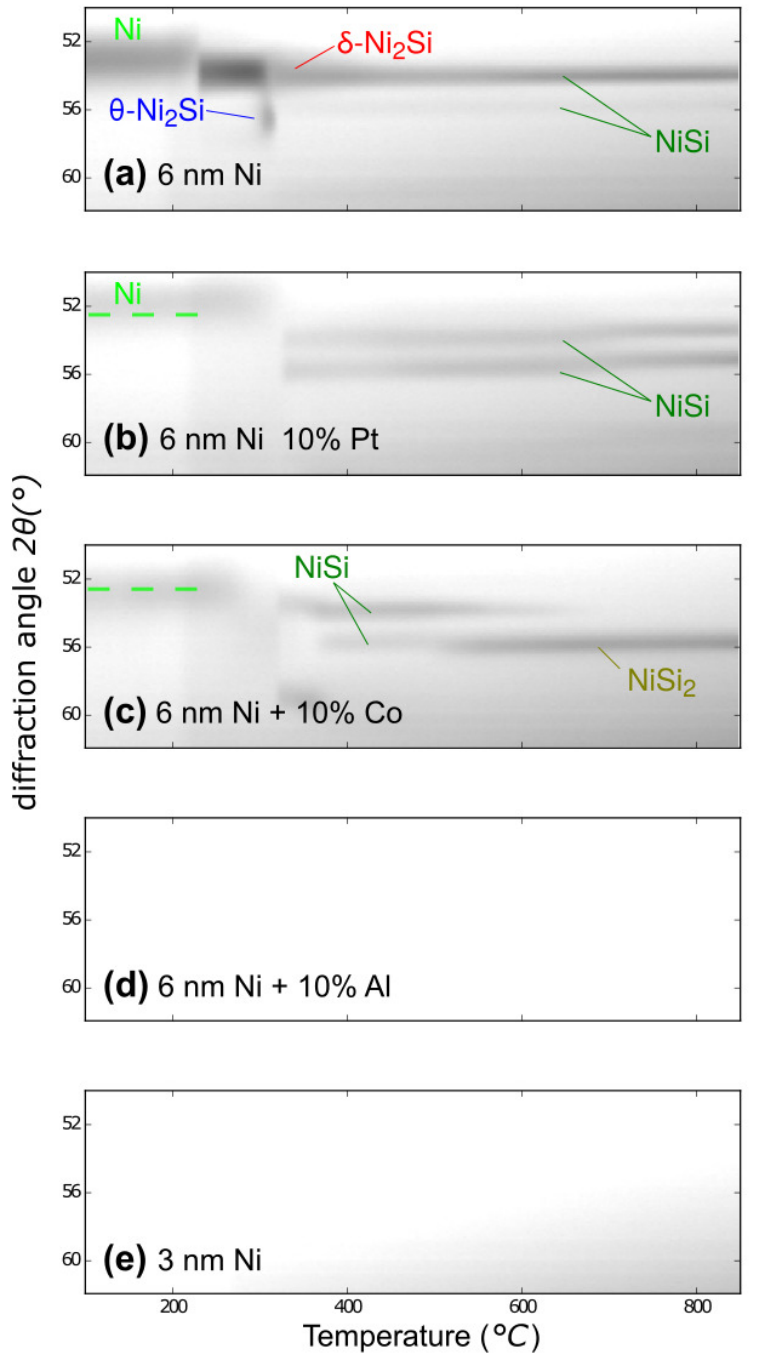

Figure 8: In situ XRD measurements of $6 \mathrm{~nm}$ thick Ni films during annealing indicate a significant difference in phase formation when comparing an unalloyed film (a) with a Pt (b), Co (c) or Al-alloyed (d) film. The latter sample shows no recognisable diffraction patterns, and as such is comparable with an unalloyed $3 \mathrm{~nm} \mathrm{Ni}$ film (e). 

is identical to the ultrathin phase sequence, as low-temperature NiSi can still be formed prior to the formation of $\mathrm{NiSi}_{2}$. This question is answered through the in situ XRD measurements for $6 \mathrm{~nm}$ thick samples, as displayed in figure 8 . From these measurements, we observe that the silicide phase sequence still includes nificantly influenced through alloying (Fig. 6). Although NiSi was observed for an unalloyed film to form at this thickness, no such diffraction patterns are observed when $\mathrm{Ni}$ was alloyed with either Co or Al. No other silicide diffraction can be observed except $\mathrm{NiSi}_{2}$, a clear indication that $\mathrm{Co}$ and $\mathrm{Al}$ alloying favours the low-temperature phase formation of $\mathrm{NiSi}_{2}$. Closer inspection of the pole figures reveals an intriguing difference in $\mathrm{NiSi}_{2}$ texture. Indeed, the sample containing 10 at.\% Co now clearly contains diffraction of epitaxial $\mathrm{NiSi}_{2}$ identified as the type- $B$ orientation. This epitaxial orientation is characterised by diffraction spots on the displayed pole figures, and can be related to type-A $\mathrm{NiSi}_{2}$ epitaxy through a rotation of $180^{\circ}$ around the $\{111\}$ direction. Furthermore, randomly-oriented $\mathrm{NiSi}_{2}$ grains are evident when alloying with Co from an overall increase in $\mathrm{NiSi}_{2}$-diffraction intensity at $\chi$ and $\phi$ angles unrelated to type A or type B epitaxy. This is illustrated in figure 7 which displays the diffraction intensities at specific locations within the reciprocal space (in contrast to the patterns displayed in Figures 3 and 6 , which were integrated over all measured $\chi$ and $\phi$ angles). Figure 7 a corresponds with type-B oriented grains, and Figure $7 \mathrm{~b}$ corresponds with randomly-oriented grains, both of which are only present in the Co-alloyed sample. Pole-figure measurement do not allow to estimate which fraction of the silicide is randomly-aligned, or along a type-A or $\mathrm{B}$ epitaxy. Al-alloying does not include the type-B features and therefore the pole figures exclude type-B epitaxy. Furthermore, these samples do not contain diffraction related to randomly-oriented $\mathrm{NiSi}_{2}$, suggesting that only type-A $\mathrm{NiSi}_{2}$ is present, similar to the case of an unalloyed Ni film thinner than $t_{c}$.

Although $\mathrm{Al}$ and Co clearly favour the low-temperature formation of $\mathrm{NiSi}_{2}$, these observations at $700{ }^{\circ} \mathrm{C}$ do not guarantee that the silicide phase formation $\mathrm{NiSi}$ at low temperatures when Co is used as alloy, while no NiSi is observed for

Films with a slightly higher equivalent $\mathrm{Ni}$ thickness, e.g. $6 \mathrm{~nm}$, are also sig- 

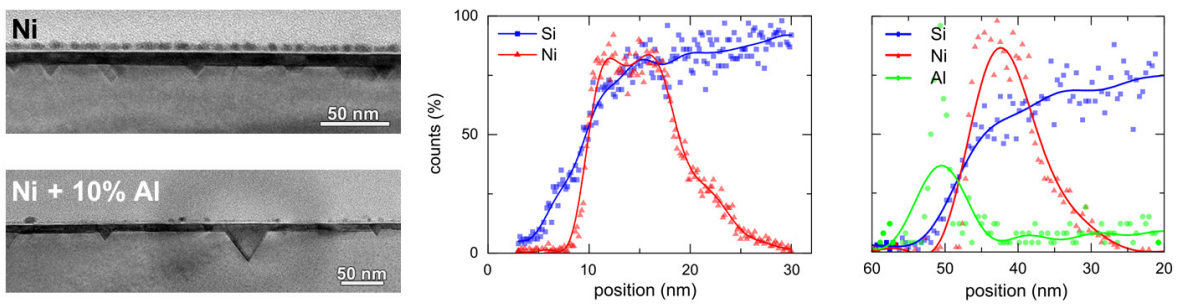

Figure 9: (left) BF-TEM images show the interface roughness of $3 \mathrm{~nm}$ Ni films without alloying, and of $6 \mathrm{~nm}$ Ni films with $\mathrm{Al}$ alloying. The EDS line scans (right) show the distribution of the alloying atoms throughout the layer thickness.

the Al-alloyed sample. We thus conclude that the critical thickness is not shifted to higher values by Co-alloying, as the phase sequence is not drastically altered and instead the transformation temperature of $\mathrm{NiSi}$ to $\mathrm{NiSi}_{2}$ is significantly reduced. On the other hand, $\mathrm{Al}$ alloying lacks all diffraction peaks through the full temperature anneal, similar to the solid-state reaction as observed for unalloyed Ni films below $t_{c}$ and indicating a promotion of the ultrathin phase sequence to thicker films. We thus conclude that the critical thickness $t_{c}$ can indeed be changed by alloying, both to higher values (Al), as well as to lower values $(\mathrm{Pt})$. Although Co-alloying does promote low-temperature formation of $\mathrm{NiSi}_{2}$, it does not change the phase sequence above the original value of $t_{c}$.

In order to evaluate the final distribution of the ternary elements, a sample of $3 \mathrm{~nm}$ unalloyed $\mathrm{Ni}$, below the critical thickness, and an Al-alloyed sample of $6 \mathrm{~nm}$ $\mathrm{Ni}$, were studied through TEM-imaging after quenching (Fig. 9). The unalloyed sample shows atomically-sharp interfaces along both the $\operatorname{Si}\{100\}$ and $\operatorname{Si}\{111\}$ directions. The latter creates triangular facets, which submerge deep into the Si substrate. Al alloying does not change this image, although Allenstein et al. report that the typical $\{111\}$ faceting disappears when alloying $20 \mathrm{~nm} \mathrm{Ni}$ films with higher $\mathrm{Al}$ concentrations (i.e. > 20 at.\%) [20]. More interesting, however, is the positioning of the alloying species throughout the silicide as determined through EDS-mapping, where almost all $\mathrm{Al}$ remains largely on the surface. 


\section{Discussion}

The critical change in phase formation in these ultra-thin films is indicative of the competitive behaviour in the low-temperature formation of either $\mathrm{NiSi}$ and $\mathrm{NiSi}_{2}$ 21]. The energetic threshold for atoms to nucleate into either of these phases can be described to be proportional to $\Delta \sigma^{3} /(\Delta H-T \Delta S)^{2}$, with $\Delta \sigma, \Delta H$ and $\Delta S$ representing the differences in interface energy, enthalpy and entropy due to the nucleation, respectively. The enthalpy of formation of the nickel silicides are comparable with one-another [19] $\delta-\mathrm{Ni}_{2} \mathrm{Si}$ : $-46.9, \mathrm{NiSi}:-42.4$, $\mathrm{NiSi}_{2}: 29.3 \mathrm{~kJ} / \mathrm{mol}$ of atoms [22]. As such, effects that change the interface energy and entropy have the potential to significantly alter the phase stability and solid-phase reaction. One can use the above equation as a framework to interpret and qualitatively rationalize the effect of ternary elements on the phase formation through entropy-considerations.

An alloy which is soluble in the forming phase will increase that phases' entropy, whereas an insoluble alloy will instead require that it should be excreted before the silicide can be formed. By consequence, soluble alloys will decrease the energy threshold for silicide nucleation whereas insoluble alloys will increase the threshold due to the required atomic transport. This argument has been used in the past to understand observed changes in ternary silicide formation for thicker films $[23,24,25,26,27,28,29$. We can thus categorise the three used alloys in three different classes, according to their solubility in $\mathrm{NiSi}$ or $\mathrm{NiSi}_{2}$. According to high-temperature $\left(>800^{\circ} \mathrm{C}\right)$ equilibrium ternary phase diagrams, $\mathrm{NiSi}_{2}$ allows relatively high incorporation of $\mathrm{Al}$ and Co atoms (20 at.\% for $\mathrm{Al}$ [30], and complete miscibility of Co instead of Ni [31]), but not of Pt. Pt is highly soluble in $\mathrm{NiSi}$, whereas the solubility of $\mathrm{Al}$ and $\mathrm{Co}$ is limited to respectively 1.5 [30] and $10 \%$ 31. On the basis of the above, one then expects $\mathrm{Pt}$ to promote the formation of $\mathrm{NiSi}$ but to delay the formation of $\mathrm{NiSi}_{2}$, and vice versa for Al. Co, however, is reasonably soluble in both phases, although to different extents. We therefore speculate that Co will not hinder the initial nucleation of NiSi grains, and so does not shift the critical thickness. However, it will 
significantly lower the nucleation temperature of $\mathrm{NiSi}_{2}$, similar to the discussion of Lavoie et al. 25] and Smeets et al. [32, so $\mathrm{NiSi}_{2}$ is formed before NiSi can severely agglomerate, resulting in a non-agglomerated film after annealing to $700^{\circ} \mathrm{C}$, as observed from the morphology evaluation of the gradient shown in figure 4. We want to point out to the reader that experiments with a higher Co concentration of 25 at.\% has been reported to increase the critical thickness [33], promoting the formation of $\mathrm{Ni}_{x} \mathrm{Co}_{1-x} \mathrm{Si}_{2}$ without first forming a mono-silicide. This difference with our experiments indicates that the influence of Co on the nickel silicide formation is different when one adds more Co to the initial film than the solubility limit of Co within NiSi (e.g. 10 at.\%). Several studies on the effect of alloying elements with higher alloying concentrations also include the formation of other phases such as $\mathrm{CoSi}$ [34] or NiAl [20, but where not observed in our in situ XRD measurements or our pole-figure XRD measurements. Our experiments with 10 at.\% Ge and Pd alloying at discrete Ni thicknesses (i.e. 2, 3153,6 and $9 \mathrm{~nm}$ ) indicate that the value of the critical thickness has decreased to below $t_{c}=3 \mathrm{~nm}$. These data are attached to this paper as supplementary information but are not further discussed in the body of this paper. As Ge and $\mathrm{Pd}$ have similar solubility properties as $\mathrm{Pt}$, these experiments corroborate the solubility argument for explaining the effect of alloying on the phase formation.

320 Luo et al. argued that the drastically altered silicide phase sequence as a function of thickness is related to the silicide-silicon interface. The energy required to facilitate this interface will become increasingly important in the total energy balance when going to thinner films. Therefore, epitaxial $\mathrm{NiSi}_{2}$ is expected to be favoured on a non-epitaxial phase such as NiSi. One can argue that the interfacial energy of the silicide-silicon interface will also be influenced through alloying. Discussions based on interface energy are not straightforward, due to a lack on experimental data on the interface energy. More recently, Kousseifi et al [35] could quantitatively determine the interface energy for Pt-alloyed $\mathrm{NiSi}$ and $\theta-\mathrm{Ni}_{2} \mathrm{Si}$. However, different alloys, or different alloying concentration, can alter the preferential orientation of silicides or change the interface energy through accumulation at the grain-boundaries. As a consequence, we do not 


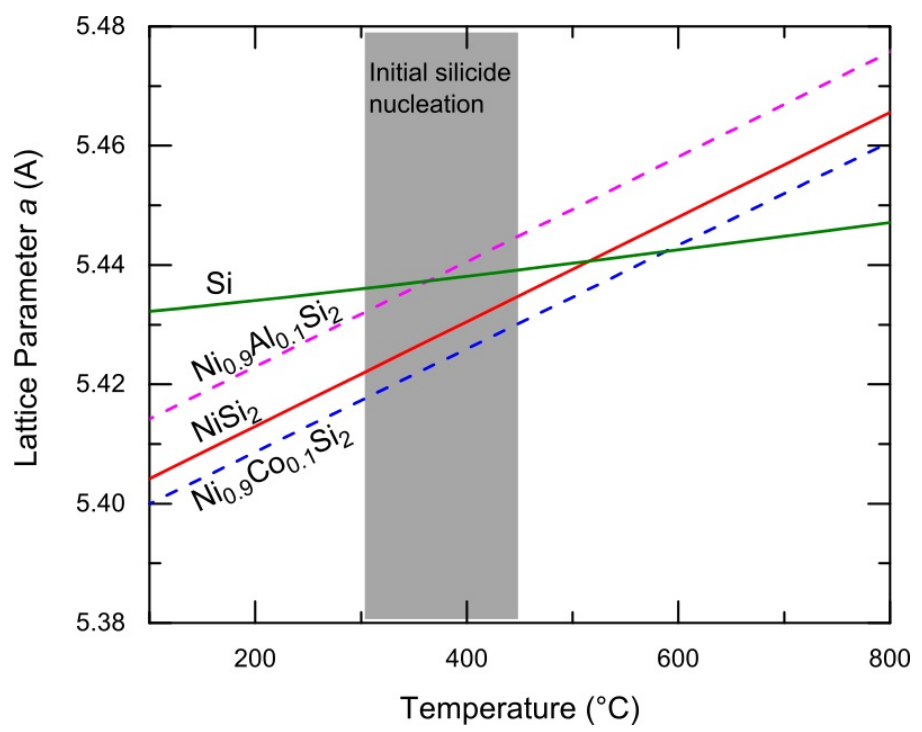

Figure 10: Evolution of the lattice parameters of $\mathrm{Si}$ and $\mathrm{NiSi}_{2}$. The influence of the alloyed elements was estimated based upon their room-temperature lattice parameter and by assuming a similar coefficient of thermal expansion.

have access to the necessary information to evaluate the effect of the interface energy on the energetic competation between $\mathrm{NiSi}$ - or $\mathrm{NiSi}_{2}$-formation. Further study that focuses on the interface, and the interphase-interface energy should prove valuable to the field. Nevertheless, we rely on the interface-energy to discuss the significant increase in randomly-aligned $\mathrm{NiSi}_{2}$ grains upon Co-alloying (Figure 7). In the ultra-thin phase formation regime, the formation of epitaxial type-A $\mathrm{NiSi}_{2}$ requires an almost perfect lattice match across the interface during initial nucleation and subsequent growth. Such a crystal match is indeed possible due to the same crystal lattice and similar lattice parameter between $\mathrm{NiSi}_{2}$ and the Si substrate. The lattice constant of $\mathrm{NiSi}_{2}$ differs only $0.46 \%$ at room temperature, and due to the different thermal expansion coefficients ( $\alpha=$ $16.25 \times 10^{-6} \mathrm{~K}^{-1}$ for $\mathrm{NiSi}_{2}$ [36] and $\alpha=2.55 \times 10^{-6} \mathrm{~K}^{-1}$ for $\mathrm{Si}$ [37]), an exact lattice match is expected during the heating process around $520^{\circ} \mathrm{C}$ (Fig. 10). However, due to the smaller lattice parameter of $\mathrm{CoSi}_{2}$, a delay in lattice-match is expected during the heating process when using Co as an alloying element. 


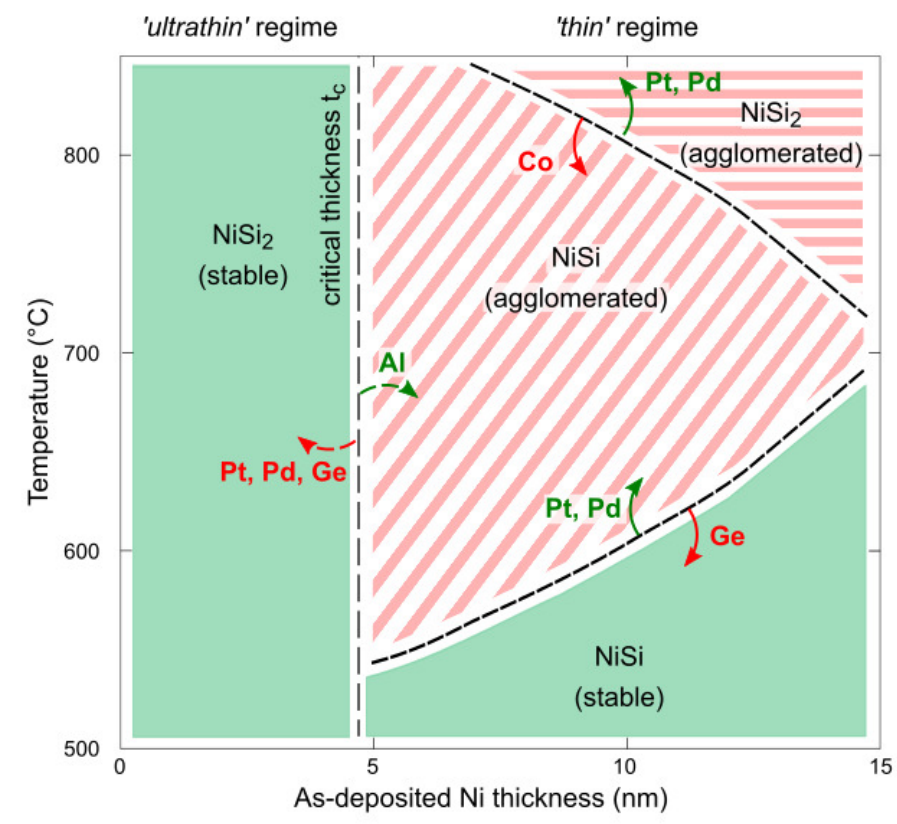

Figure 11: Overview of the thermodynamic and morphological stable regimes for nickel silicides as a function of thickness and annealing temperature. Alloying the initial Ni film with low $(<10 \%)$ concentration of $\mathrm{Al}, \mathrm{Co}, \mathrm{Ge}, \mathrm{Pd}, \mathrm{Pt}$ are known to influence these degradation temperatures.

On the other hand, $\mathrm{Al}$ is reported to increase the lattice parameter of $\mathrm{NiSi}_{2}$, and as such, lowers the temperature at which we expect an exact lattice match. From in situ sheet resistance data [9, we expect the initial nucleation of $\mathrm{NiSi}_{2}$ grains in the ultrathin phase formation regime between $300-400^{\circ} \mathrm{C}$. By consequence, we propose that Al-alloying will require less strain or interfacial defects at nucleation to compensate the difference in the lattice parameter than Coalloying. In other words, on the basis of lattice matching arguments one would also conclude that alloying with $\mathrm{Al}$ is expected to lower the energy threshold to form epitaxial $\mathrm{NiSi}_{2}$ at low temperatures.

Our results can be put into the broader context of stable nickel silicide contacts. The degradation of nickel silicides is discussed ever since NiSi was first considered as a contact material for the transistors' source and drain regions. 
The stability of a NiSi film is challenged through (1) agglomeration and (2)

\section{Conclusions}

The Ni-Si solid-state reaction was studied in the sub-15 nm Ni thickness regime. The morphological stability of the formed silicides indicates a critical thickness $t_{c}=4.8 \mathrm{~nm}$ that separates the 'regular' phase sequence, which forms agglomerating NiSi, from the more stable 'ultrathin' phase sequence, which forms epitaxial $\mathrm{NiSi}_{2}$ at low temperatures. Thickness gradient libraries were fabricated to investigate the effect of ternary elements (10 at.\% Al, Co and Pt) on the value 
of $t_{c}$ as a function of thickness in a semi-continuous fashion. We found that $t_{c}$ can be increased or decreased at will, by the addition of $\mathrm{Al}\left(t_{c}=8.0 \mathrm{~nm}\right)$ and $\mathrm{Pt}$ $\left(t_{c}=2.6 \mathrm{~nm}\right)$ as alloy. Co did not change the phase sequence, did not display an abrupt critical thickness. It did, however, alter the transformation temperature of $\mathrm{NiSi}$ into $\mathrm{NiSi}_{2}$ which resulted in a rough but non-agglomerating silicide film. Several energetic parameters are discussed to understand the observed shift in relation to mixing entropy and interface energy. This study shows that the solid-phase reaction of silicides exhibits drastic non-linear behaviour upon thickness scaling, and which can be influenced and controlled by altering the as-deposited composition.

\section{Supplementary Materials}

All polefigures displayed in this article are oriented with fixed position in reference to the Si substrate, as displayed in the supplementary material online.

Overview of in situ XRD measurements and SEM micrographs of $10 \mathrm{~nm}$ $\mathrm{Ni}$, alloyed with 10 at.\% Ge and Pd are also displayed in the supplementary material.

\section{Acknowledgement}

The authors acknowledge the FWO Vlaanderen, the Hercules foundation and BOF-UGent (GOA 01G01513) for providing financial support for this work. This research used resources of the National Synchrotron Light Source, a U.S. Department of Energy (DOE) Office of Science User Facility operated for the DOE Office of Science by Brookhaven National Laboratory under Contract No.

DE-AC02-98CH10886.

\section{References}

[1] C. Lavoie, F. d'Heurle, C. Detavernier, C. Cabral, Towards implementation of a nickel silicide process for CMOS technologies, Microelectronic Engineering 70 (2) (2003) 144-157. 
[2] A. Lauwers, J. A. Kittl, M. J. Van Dal, O. Chamirian, M. A. Pawlak, M. de Potter, R. Lindsay, T. Raymakers, X. Pages, B. Mebarki, et al., Ni based silicides for 45nm CMOS and beyond, Materials Science and Engineering: B 114 (2004) 29-41.

[3] D. Deduytsche, C. Detavernier, R. Van Meirhaeghe, C. Lavoie, Hightemperature degradation of NiSi films: Agglomeration versus $\mathrm{NiSi}_{2}$ nucleation, Journal of Applied Physics 98 (3) (2005) 033526.

[4] J. Kittl, K. Opsomer, C. Torregiani, C. Demeurisse, S. Mertens, D. Brunco, M. Van Dal, A. Lauwers, Silicides and germanides for nano-CMOS applications, Materials Science and Engineering: B 154 (2008) 144-154.

[5] D. Mangelinck, J. Dai, J. Pan, S. Lahiri, Enhancement of thermal stability of NiSi films on (100) Si and (111) Si by Pt addition, Applied Physics Letters 75 (12) (1999) 1736-1738.

[6] Z. Zhang, S. Zhang, B. Yang, Y. Zhu, S. M. Rossnagel, S. Gaudet, A. J. Kellock, J. Jordan-Sweet, C. Lavoie, Morphological stability and specific resistivity of sub-10 nm silicide films of $\mathrm{Ni} \mathrm{Pt}_{x}$ on Si substrate, Applied Physics Letters 96 (2010) 071915.

[7] N. Breil, C. Lavoie, A. Ozcan, F. Baumann, N. Klymko, K. Nummy, B. Sun, J. Jordan-Sweet, J. Yu, F. Zhu, et al., Challenges of nickel silicidation in CMOS technologies, Microelectronic Engineering 137 (2015) 79-87.

[8] R. Tung, J. Poate, J. Bean, J. Gibson, D. Jacobson, Epitaxial silicides, Thin Solid Films 93 (1) (1982) 77-90.

[9] K. De Keyser, C. Van Bockstael, R. Van Meirhaeghe, C. Detavernier, E. Verleysen, H. Bender, W. Vandervorst, J. Jordan-Sweet, C. Lavoie, Phase formation and thermal stability of ultrathin nickel-silicides on $\mathrm{Si}$ (100), Applied Physics Letters 96 (17) (2010) 173503.

[10] Z. Zhang, B. Yang, Y. Zhu, S. Gaudet, S. Rossnagel, A. J. Kellock, A. Ozcan, C. Murray, P. Desjardins, S. Zhang, et al., Exploitation of a self- 
limiting process for reproducible formation of ultrathin $\mathrm{Ni}_{1-x} \mathrm{Pt}_{x}$ silicide films, Applied Physics Letters 97 (25) (2010) 252108.

[11] J. Fouet, M. Texier, M.-I. Richard, A. Portavoce, D. Mangelinck, C. Guichet, N. Boudet, O. Thomas, Silicide formation during reaction between Ni ultra-thin films and $\mathrm{Si}$ (001) substrates, Materials Letters 116 (2014) 139-142.

[12] L. Knoll, Q. Zhao, S. Habicht, C. Urban, B. Ghyselen, S. Mantl, Ultrathin $\mathrm{Ni}$ silicides with low contact resistance on strained and unstrained silicon, IEEE Electron Device Letters 31 (4) (2010) 350-352.

[13] L. Knoll, Q. Zhao, R. Luptak, S. Trellenkamp, K. Bourdelle, S. Mantl, 20nm Gate length Schottky MOSFETs with ultra-thin NiSi/epitaxial $\mathrm{NiSi}_{2}$ source/drain, Solid-State Electronics 71 (2012) 88-92.

[14] X. Gao, J. Andersson, T. Kubart, T. Nyberg, U. Smith, J. Lu, L. Hultman, A. J. Kellock, A. Zhang, C. Lavoie, et al., Epitaxy of ultrathin $\mathrm{NiSi}_{2}$ films with predetermined thickness, Electrochemical and Solid-State Letters 14 (7) (2011) H268-H270.

[15] C. Detavernier, J. Jordan-Sweet, C. Lavoie, Texture of NiSi films on Si (001),(111), and (110) substrates, Journal of Applied Physics 103 (11) (2008) 113526.

[16] B. De Schutter, K. De Keyser, C. Detavernier, Visualization and classification of epitaxial alignment at hetero-phase boundaries, Thin Solid Films 599 (2016) 104-112.

[17] B. De Schutter, K. De Keyser, C. Lavoie, C. Detavernier, Texture in thin film silicides and germanides: A review, Applied Physics Reviews 3 (3) (2016) 031302.

[18] P. Schlossmacher, D. Klenov, B. Freitag, H. Von Harrach, Enhanced detection sensitivity with a new windowless XEDS system for AEM based on silicon drift detector technology, Microscopy Today 18 (04) (2010) 14-20. 
[19] S. Gaudet, C. Coia, P. Desjardins, C. Lavoie, Metastable phase formation during the reaction of Ni films with $\mathrm{Si}(001)$ : The role of texture inheritance, Journal of Applied Physics 107 (9) (2010) 093515.

[20] F. Allenstein, L. Budzinski, D. Hirsch, A. Mogilatenko, G. Beddies, R. Grötzschel, H. Hinneberg, Influence of $\mathrm{Al}$ on the growth of $\mathrm{NiSi}_{2}$ on Si (001), Microelectronic Engineering 82 (3) (2005) 474-478.

[21] J. Luo, Z. Qiu, C. Zha, Z. Zhang, D. Wu, J. Lu, J. Åkerman, M. Östling, L. Hultman, S. Zhang, Surface-energy triggered phase formation and epitaxy in nanometer-thick $\mathrm{Ni}_{1-x} \mathrm{Pt}_{x}$ silicide films, Applied Physics Letters $96(3)$.

[22] M. E. Schlesinger, Thermodynamics of solid transition-metal silicides, Chemical Reviews 90 (4) (1990) 607-628.

[23] C. Detavernier, R. Van Meirhaeghe, F. Cardon, K. Maex, Influence of mixing entropy on the nucleation of $\mathrm{CoSi}_{2}$, Physical Review B 62 (18) (2000) 12045.

[24] A. Lauwers, M. De Potter, O. Chamirian, R. Lindsay, C. Demeurisse, C. Vrancken, K. Maex, Silicides for the 100-nm node and beyond: Cosilicide, $\mathrm{Co}(\mathrm{Ni})$-silicide and $\mathrm{Ni}$-silicide, Microelectronic Engineering 64 (1) (2002) 131-142.

[25] C. Lavoie, C. Detavernier, C. Cabral, F. dHeurle, A. Kellock, J. JordanSweet, J. Harper, Effects of additive elements on the phase formation and morphological stability of nickel monosilicide films, Microelectronic Engineering 83 (11) (2006) 2042-2054.

[26] J. Demeulemeester, D. Smeets, C. Comrie, N. Barradas, A. Vieira, C. Van Bockstael, C. Detavernier, K. Temst, A. Vantomme, On the growth kinetics of Ni (Pt) silicide thin films, Journal of Applied Physics 113 (16) (2013) 163504. 
[27] J. Toinin, A. Portavoce, M. Texier, M. Bertoglio, K. Hoummada, First stages of $\mathrm{Pd} / \mathrm{Ge}$ reaction: Mixing effects and dominant diffusing species, Microelectronic Engineering.

[28] A. Schrauwen, J. Demeulemeester, D. Deduytsche, W. Devulder, C. Detavernier, C. Comrie, K. Temst, A. Vantomme, Ternary silicide formation from Ni-Pt, Ni-Pd and Pt-Pd alloys on Si (100): Nucleation and solid solubility of the monosilicides, Acta Materialia 130 (2017) 19-27.

[29] A. Schrauwen, K. Van Stiphout, J. Demeulemeester, B. De Schutter, W. Devulder, C. Comrie, C. Detavernier, K. Temst, A. Vantomme, The role of composition and microstructure in $\mathrm{Ni}-\mathrm{W}$ silicide formation and low temperature epitaxial $\mathrm{NiSi}_{2}$ growth by premixing $\mathrm{Si}$, Journal of Physics D: Applied Physics 50 (6) (2017) 065303.

[30] K. Richter, K. Chandrasekaran, H. Ipser, The Al-Ni-Si phase diagram. Part II: phase equilibria between 33.3 and 66.7 at.\% Ni, Intermetallics $12(5)(2004) 545-554$.

[31] J. Van Beek, P. Oberndorff, A. Kodentsov, F. Van Loo, Interactions in the Co-Ni-Si system at 800 C, Journal of Alloys and Compounds 297 (1) (2000) 137-143.

[32] D. Smeets, J. Demeulemeester, K. De Keyser, D. Deduytsche, C. Detavernier, C. Comrie, C. Theron, C. Lavoie, A. Vantomme, Nucleation and diffusion during growth of ternary Co1- xNixSi2 thin films studied by complementary techniques in real time, Journal of Applied Physics 104 (9) (2008) 093533.

[33] Z. Zhu, X. Gao, Y. Piao, C. Hu, Z. Qiu, Z.-B. Zhang, D. Wu, S.-L. Zhang, Phase formation and film morphology of ultrathin $\mathrm{Co}_{1-x} \mathrm{ni}_{x} \mathrm{Si}_{2}$ films, Journal of Vacuum Science \& Technology A: Vacuum, Surfaces, and Films 30 (5) (2012) 050602. 
${ }_{525}$ [34] Y. Piao, Z. Zhu, X. Gao, A. Karabko, C. Hu, Z. Qiu, J. Luo, Z.-B. Zhang, S.-L. Zhang, D. Wu, Extensive raman spectroscopic investigation of ultrathin co1xnixsi2 films grown on si(100), Journal of Vacuum Science \& Technology A: Vacuum, Surfaces, and Films 30 (4) (2012) 041511. doi:10.1116/1.4726295.

${ }_{530}$ [35] M. El Kousseifi, K. Hoummada, T. Epicier, D. Mangelinck, Direct observation of $\mathrm{NiSi}$ lateral growth at the epitaxial $\theta-\mathrm{Ni}_{2} \mathrm{Si} / \mathrm{Si}(100)$ interface, Acta Materialia 99 (2015) 1-6.

[36] D. Smeets, G. Vanhoyland, J. D'Haen, A. Vantomme, On the thermal expansion coefficient of cosi2 and nisi2, Journal of Physics D: Applied Physics 42 (23) (2009) 235402.

[37] Y. Okada, Y. Tokumaru, Precise determination of lattice parameter and thermal expansion coefficient of silicon between 300 and $1500 \mathrm{k}$, Journal of Applied Physics 56 (2) (1984) 314-320.

[38] A. Derafa, G. Tellouche, K. Hoummada, A. Bouabellou, D. Mangelinck, Effect of alloying elements mo and w on ni silicides formation, Microelectronic Engineering 120 (2014) 150-156.

[39] Y. Setiawan, P. Lee, C. Tan, K. Pey, Effect of Ti alloying in nickel silicide formation, Thin Solid Films 504 (1) (2006) 153-156.

[40] D. Deduytsche, C. Detavernier, R. Van Meirhaeghe, J. Jordan-Sweet, C. Lavoie, Formation and morphological stability of NiSi in the presence of W, Ti, and Ta alloying elements, Journal of Applied Physics 101 (4) (2007) 044508 .

[41] D. Deduytsche, C. Detavernier, R. Van Meirhaeghe, J. Jordan-Sweet, C. Lavoie, Formation and stability of NiSi in the presence of Co and Fe alloying elements, Journal of Vacuum Science \& Technology B 26 (6) (2008) 1971-1977. 
[42] T. Yang, G. Luo, E. Chang, T. Yang, H. Tseng, C. Chang, Study of nickel silicide contact on $\mathrm{Si} / \mathrm{Si}_{1-x} \mathrm{Ge}_{x}$, IEEE Electron Device Letters 24 (9) (2003) 544-546.

555 [43] P. Besser, P. King, E. Paton, S. Robie, Ge effects on silicidation, Microelectronic Engineering 82 (3) (2005) 467-473. 